\title{
A Survey of the State of Cloud Computing in Healthcare
}

\author{
Sanjay P. Ahuja ${ }^{1}$, Sindhu Mani ${ }^{1} \&$ Jesus Zambrano ${ }^{1}$ \\ ${ }^{1}$ School of Computing, University of North Florida, Jacksonville, USA \\ Correspondence: Sanjay P. Ahuja, School of Computing, University of North Florida, Jacksonville, FL 32224, \\ USA.E-mail: sahuja@unf.edu
}

Received: July 26, 2012 Accepted: September 3, 2012 Online Published: September 19, 2012

doi:10.5539/nct.v1n2p12 URL: http://dx.doi.org/10.5539/nct.v1n2p12

\begin{abstract}
Analysts, researchers and organizations alike seem to agree that cloud computing will be a defining trend in the coming decade impacting wide range of businesses and how those businesses are practiced. Large technology companies are already investing millions of dollars in building infrastructure, services, tools and applications to facilitate cloud computing for consumers, organizations and businesses to use and take advantage. It remains to be seen how cloud computing will impact the healthcare business since it is very diverse, complex and unique and presents several challenges such as protecting members health records in addition to following HIPAA guidelines set by federal compliance regulations. In addition to these the rising cost of healthcare solutions is another major concern. Efforts are being made to reduce these costs for consumers and IT will play a big role in achieving it and also improving clinical and quality outcomes for patients. It will be very interesting to see how cloud computing will address and contribute towards these issues in the healthcare industry. The purpose of this paper is to explore the current state and trends of cloud computing in healthcare.
\end{abstract}

Keywords: healthcare, cloud, security, HIPAA, EHR

\section{Introduction}

The advent of cloud computing and its business models have been some of the biggest changes impacting not only the computer industry but also several others. It is predicted that around $80 \%$ of the businesses from today's world will be moved to the cloud by the year 2020 (Kuttikrishnan, 2011). Organizations that do not have enough resources to invest and build infrastructure and platforms to deploy their applications can now take advantage of the cloud services to suit their specific needs. With the pay-as-you-go model the consumers pay for what they use and how much they use. The cloud has infrastructure built so consumers can deploy and run their applications. They also have different platforms with multiple operating systems so consumers can build, test, and deploy their applications in virtual servers. In addition, the cloud provides highly scalable environment to effectively handle the load. Since the infrastructure and platforms are already built and are available, consumers and organizations can save a lot since they don't have to invest in them or maintain them. Some of the bigger organizations are investing in building their own private clouds by leveraging their existing infrastructure and tools.

Since the real life businesses and organizations normally build applications in quite a complex environment that involves networking, security, physical servers, firewalls etc. and transactions worth millions of dollars, they expect same or higher level of service provided by the cloud service providers and the cloud so their data is protected and the businesses are not negatively impacted. More and more tools are being built on the Internet that is important to run a business. Healthcare industry has been one of the businesses that traditionally repelled outsourcing mainly because of the security and privacy concerns. The member's privacy and medical records are highly sensitive and the companies spend millions in protecting them while following the federal regulated guidelines. However, rising medical costs might change the way this business is performed.

\section{Moving to the Cloud}

Cloud computing can play a critical role in containing healthcare integration costs, optimizing resources and ushering in a new era of innovations. Current trends aim towards accessing information anytime, anywhere, which can be achieved when moving healthcare information to the cloud. This new delivery model can make healthcare more efficient and effective, and at a lower cost to technology budgets (Horowitz, 2011), but also, it brings certain obstacles due to concerns related with safeguarding confidential patient information and 
compliance with key regulations such as HIPAA. Despite these security and privacy risks, healthcare organizations can certainly take advantage of cloud computing solutions and bring tremendous benefits such as help to improve patient quality of service and reduce overall healthcare costs (Muir, 2011).

One of the key benefits will be the ability to exchange data between disparate systems. This capability is something healthcare IT is desperately needs. For instance, cloud computing can support healthcare organizations to share information such as EHR, doctor's references, prescriptions, insurance information, test results stored across different information systems. This is already happening in the radiological area, where many institutions have moved to the cloud to lower their storage costs and facilitate the exchange of images (Terry, 2012).

Nine times out of 10 , moving offsite is going to be more financially attractive than trying to build out your own organization in-house (Horowitz, 2011). When offloading healthcare organizations the IT staff required to maintain network availability, security updates, backups and others can be free up to attend to more critical tasks in an efficient and cost-effective manner (Muir, 2011). In addition, more reliable and powerful infrastructures will be available pay-as-you-go and it can be managed dynamically.

Another issue that needs addressing is the potential candidate to which to move. The last thing an organization wants is to move a lot of their processing to a third party and find out that it is going to be acquired by a company who the organization doesn't necessarily want to be a partner with or just is going to dissolve (Horowitz, 2011). The best cloud provide is one which that has been in the healthcare business already, so they know about HIPAA compliance and issues that go along with it. In addition, a well-defined plan and the right tools can support the transition to cloud-based systems to take advantage of the benefits it brings (Muir, 2011).

Cloud computing has given opportunities for clinics, hospitals, insurance companies, pharmacies, and other healthcare companies to agree in collaborating between them and share healthcare information to offer better quality of service and reduce costs. Looking over the changes occurring in the market, it appears that cloud-based systems will likely become the norm in healthcare once all the challenges it brings are overcome.

\section{Considerations for Healthcare Facilities before Moving to the Cloud}

Healthcare organization have been dealing with growing amounts of electronic records and digital images, would seem a good fit for cloud storage services. Among the reasons for this sudden interest in cloud-based computing and communications are the need for collaboration among the increasing number of remote and mobile workers, several office locations, a desire to improve patient quality of service and the ever present goals of improving operational excellence while driving down technology equipment and healthcare management costs (Whitemore, 2012).

To help healthcare organizations decide whether they ready to move to the cloud there are a few important points to consider.

\subsection{Distributed vs. Centralized Facilities}

Depending on how the health organization operates - whether their IT infrastructure are distributed between their medical facilities or in a datacenter, moving to the cloud would help communications, applications, and collaboration between the health organizations. This approach reduce the need for IT staff in each facility satellite station, as well as to reduce IT budget.

\subsection{Prepare the Infrastructure}

Most of the current networks at healthcare facilities and their multiple office locations were built years ago. Adopting cloud-based systems, an inadequate infrastructure can quickly collapse due the high network traffic by up-tick in voice, video and data traffic applications deal with. In addition, deploying a MPLS (Multiprotocol Label Switching) will keep a reliable internet connection due the multiple connections and redundancies; if the main carrier's connection goes down, it will automatically switch over to one of the others, providing the high-level business reliability and disaster recovery that many of the healthcare facilities don't have today.

\section{Healthcare Security Issues with Cloud Computing}

While the cloud appears to present several benefits it also appears to present special risks to healthcare organizations with respect to privacy and security. It is very important that the cloud service providers fully understand these security concerns in public clouds and address them as the healthcare organization comply with HIPAA privacy rules set by federal regulations. Guaranteeing security on storing medical health records and safeguarding them will be their most important jobs since cloud computing seems to be the future for this purpose (Allen, 2011). This health records information also needs to be made centrally accessible while still 
supporting these privacy guidelines. Cloud Security Alliance (Cloud Security Alliance, 2010) has published a list of best practices that should be implemented in the cloud to achieve maximum security in the Service as a Platform (SaaS) and Platform as a Service (PaaS) models. The alliance advises that following these security procedures will provide robust security against potential threats in both private and public clouds. It suggests implementing several layers of authentication to adequately secure health data records such as authenticating user login, administration capabilities to assign appropriate privileges to the users and groups, enforcing strong passwords creation and encryption, encrypted data exchange (Cloud Security Alliance, 2010).

In addition, consider federated authentication (Cloud Security Alliance, 2010) which delegates authentication to the organizations that uses SaaS. As a user the healthcare organizations must ensure that their cloud provider enforces these security measures. Because of the amount of personal information available in the health records it could be a target for hackers and malicious users and use it their benefits. Insecure APIs and loose interface can be easy targets for these users. Breach notification enforcement is another security practice, which is a law in some states.

The backup services and disaster recovery policies definitely appear to be more robust and cloud providers are equipped than the local data centers that organizations maintain. Bigger cloud providers such Google App Engines and Amazon EC2 create and manage several copies of the data at different locations and data retrieval processes. So in the event of any data loss it can be restored without much damage. Data can be more easily and widely available and more consistent than that of privately maintained data centers. In that view the cloud data storages can be considered for persistent data storages by the health organizations. Designing and implementing community cloud model can be another approach to create more interconnected network of the healthcare providers and organizations (Raut, 2011) to mitigate some security concerns. The cloud infrastructure can be shared across payers and other healthcare entities and demand specific security needs and policies. This might create applications on infrastructure that will help in sharing the information in secured environment and quickly. This model may be a little bit different from public cloud model but it will allow the organizations to address security changes specific to their needs. Since the data, images and other records will be available within the community physicians, pharmacies, payers can perform tasks such as counseling, diagnose faster there reducing costs for everyone involved.

\section{Applications of Cloud Computing in Healthcare}

The ecosystem of the healthcare system is vast, diverse and highly complex that includes health insurance companies, hospital and physician networks, laboratories, pharmacies, patients and other entities (Wan, Greenway, Harris, \& Alter, 2010). And all these must work within several governmental regulations (Raut, 2011) In order to for this ecosystem to perform effectively and fast it is critical that some important information is shared quickly and accurately between them confidentially and in a secured way between these entities. Securing the patient's information is considered very sensitive and privacy concerns in the healthcare industry. Probably one of the reasons, which has negatively impacted the progress of healthcare moving into the cloud. Sharing of it must be handled with innovative technologies and tools when it comes to cloud. There are however lot of other data, information and services that can undoubtedly benefit by collaboration from using the cloud as they potentially span between cities, states and even countries. In the current scenario it appears that private clouds will be implemented first due to security concerns and then move into public infrastructure (Wan, Greenway, Harris, \& Alter, 2010). May be it will be a good idea to first layout the top priorities of the healthcare industry and then evaluate which cloud computing aspects can be effectively applied to benefit them. In today's world rising health care costs, quality of services provided to the patient and customers, privacy, data security \& integrity, and disaster recovery appear to be the top priorities (Wan, Greenway, Harris, \& Alter, 2010; Cloud Security Alliance, 2010). Some of the inherent features such as scalable infrastructure, data centers for providing persistent data, security models, fast access to information etc. can be leveraged so some of these priorities can be addressed.

\subsection{Infrastructure and Dynamic Scalability}

As more and more customer base increases in the healthcare industries, healthcare solutions providers also grow and so does the business. As the business grows the organizations invest heavily in more number of computing capacity and IT resources to tackle the growing need. These computing resources are provisioned to serve quite complex and dynamic environment. With cloud computing the organizations now have an alternative to address this issue. The cloud provides Infrastructure-as-a-Service and Platform-as-a-Service business models where organizations can use the existing infrastructure or customize to suit their business and needs. More servers can be added or removed as needed within a short amount of time. Instead of storing the data like hospital 
information, physician networks, pharmacies, their locations etc. in the private on-site servers they can shift them to the cloud based data centers for storage. Keeping up with the software updates will also be primarily handled by the vendor. This will eventually free-up several recourses and reduce the cost of maintaining them for the healthcare solutions providers. Since the cloud provides scalable infrastructure, the organizations may be able to better adjust and optimize their resource capacity planning. For some of the clinics that are small to medium sized and can't afford large IT investments and staff can take great advantage from cloud computing with their cost effective fee structure and low cost maintenance. An example for this is Amazon's S3 that provides scalable storage architecture (Kuo, 2011). eHealth cloud (Korea IT Times, 2010) is being built by Telstra and the Royal Australian College of General Practitioners and is expected to host healthcare applications such as "clinical software, decision-support tools for diagnosis and management, care plans, referral tools, prescriptions, training, and other administrative and clinical services" (Korea IT Times, 2010).

\subsection{Information Sharing}

Health organizations do not function in silo. They have to constantly interact with other organizations as mentioned above. A lot of information is sent and received in order to process claims, provide customer support services, new member acquisitions, process provider requests etc. In today's world providers store the EMRs in their own databases (Rui \& Ling, 2010). If some of this information can be moved to the cloud and shared across these organizations and platforms it may lead to better and faster coordination of services and eventually more customer satisfaction. With explicit customer's consent some of the patient's information such as Electronic Health Records (EHRs), Electronic Medical Records (EMRs), Personal Health Records (PHRs), Payer Based Health Records (PBHRs) (Shimrat, 2009) etc. can be moved to the cloud and can be shared with hospitals and physicians that are located in different states or countries. Same information will be lead to fewer errors (Raut, 2011). This has huge a potential in providing faster and higher quality of service yet saving cost. Microsoft's HealthVault (Microsoft, 2012) can be very useful providing some of these benefits. The motivation behind HealthVault is to create a centralized place to store health information such as medical images, doctor's fax records, turning them into digital formats etc. It can also help connect pharmacies, labs, hospitals, clinics etc. to retrieve and use health information to their needs and provide better and faster services to patients. Consumer can use the HealthVault Connection Center to add data to their health records from their health and fitness devices such as heart rate monitors and blood pressure monitors etc. (Microsoft, 2012). Besides Microsoft, Oracle's Exalogic Elastic Cloud and Amazon's Web Services are also showing lot of promise is providing storage of health information in the cloud (Kuo, 2011).

Acumen states in HealthImaging (2010), "The US Department of Health \& Human Services' Office of the National Coordinator for Health Information Technology recently chose Acumen Solutions' cloud-based customer relationship management and project management system for the selection and implementation of EHR systems across the United States. The software enables regional extension centers to manage interactions with medical providers related to the selection and implementation of an EHR system (HealthImaging, 2010)."

In a new offering Accenture and AT\&T have designed a cloud based medical imaging service called as Accenture Medical Imaging Solution (Violino, 2011). This service is built for the medical professionals to review the medical images such as X-rays, MRI and CT scans instantly allowing them see more patients than they do today. This service will provide central management of these images so they can be reviewed, exchanged and shared by the healthcare providers in a faster and highly secured environment.

\subsection{Availability}

High availability of the cloud services can only help the health organizations to provide uninterrupted services with minimum downtimes. The cloud can be programmatically controlled so that near real-time scalability capabilities can be achieved. Clusters can be created with several nodes to achieve high availability (Kupferman, Silverman, Jara, \& Browne, 2009). And since the resources are fixed at the beginning of the computation the applications can be scaled up or scaled down as the requirement to adjust the workload arises. There are also predictions that managing healthcare applications in the cloud will make them more widely available and at all times. This could drastically reduce the costs of maintenance. In-depth understanding of the healthcare security and privacy concerns could be the first step in moving the healthcare applications into the cloud (Rui \& Ling, 2010).

\subsection{Cloud Monitoring Tools}

In addition to rise of cloud computing several third party vendors are developing tools that can help monitor the services provided in the cloud. Healthcare organizations work with these vendors in customizing these tools with appropriate security features to suit their businesses. There are several third party vendors that are on the rise that 
provide monitoring and cloud management tools such as Cloudkick (Rackspace, 2011), LogicMonitor (LogicMonitor, 2012), Pandora FMS (Pandora, 2011) etc. More importantly several vendors provide tools that only provide monitoring of the server or the service up-time (Barry, 2011). Amazon Web Services provides it own monitoring tools on the EC2 instances that customer take advantage of.

\section{Benefits of Adopting Cloud for Healthcare Organizations}

Cloud computing brings a new business model which enables several advantages that would benefit the general healthcare community. By adopting the cloud in medical services both patients and healthcare organizations would obtain a huge benefit in patient's quality of service, collaboration between healthcare organizations as well as reductions in IT cost in healthcare companies.

This collaborative approach enables healthcare services to interoperate between them in order to offer a faster and efficient response helping to improve the patient quality of service through sharing information across healthcare organizations. Therefore, hospitals, clinics, imaging centers, pharmacies and insurance companies can efficiently share patient's medical records, prescription information, $X$ rays, test results, physician's references, physicians availability, etc. that can be accessed anywhere and everywhere by authorized entities. All this information would be used for making decisions, obtaining better diagnosis and treatments to yield better results, scheduling physician's appointments, speeding insurance approval, etc. which highly improves patient's quality of service.

When moving to the cloud, there is also a very important beneficial factor for healthcare organizations, which is the IT costs. By adopting the cloud model, all the IT processes will be migrated to the remote cloud-computing infrastructure where all the processes will be performed and stored. The new "pay-as-you-go" model allows organizations to pay only for what they use; therefore, there is no reason for acquiring expensive hardware infrastructure, software licenses or to keep/train in-site staff for maintenance, security, replications because the cloud computing providers takes care of them.

Human life is priceless, and medical resources are limited (Wang, 2010) therefore, healthcare services adopted in cloud providers match a cost-effective concept where patients and health organizations take advantages of this new technology by improving patients quality of service through a distributed high-integrated platform (Wang, 2010), coordinating of medical process as well as reducing IT infrastructure investment or maintenance costs which leads to a better healthcare environment.

\section{Global Market for Cloud Computing in Healthcare}

Cloud is not going anywhere as many IT giants like Amazon, Google, Microsoft and many others are heavily investing in it by building large data centers to support it and help organizations take benefit from it. They are collaborating with their health partners to build medical record services (Kabachinski, 2011). Microsoft collaborated with Kaiser Permanente to Microsoft HealthVault applications (Kabachinski, 2011). Its main focus is to store health and fitness information such as in one location so it can organized and shared easily across healthcare entities including patients. Google Health uses cloud services to get health records from Cleveland Clinic's MyChart program (Kabachinski, 2011).

For many healthcare organizations, the cost effective models of the cloud are already forcing them to review their current business practices including risks and benefits to check how they can adopt cloud (Webb, 2012). The faster these businesses adopt cloud, faster will they will achieve greater efficiencies and provide unprecedented sharing capabilities between the healthcare organizations and patients alike.

According to marketsandmarkets.com (M\&M) research and survey, around $32 \%$ of the healthcare organizations are already using some cloud-based applications. Around $75 \%$ of the organizations that are currently not using any cloud applications responded that they are considering cloud adoption within next three to five years (Blaisdell, 2012). According to their research public cloud model will be adopted first followed by hybrid model and eventually to private cloud model for their strategic businesses.

These findings appear to be consistent with the survey conducted by Accenture in early 2010. They also indicate that early adopters of the cloud within the healthcare industry will have competitive advantage in terms of cost and effectiveness compared to the ones that lag behind (Wan, Greenway, Harris, \& Alter, 2010).

\section{Challenges of Cloud Computing in Health Care}

The slow adoption of the Cloud Computing model in the health field is mostly due to two important concerns associated with security and interoperability. Those issues need to be addressed in order to overcome doubts when moving to cloud and taking advantage of all the solutions and improvements it brings. 


\subsection{Security Concerns}

Healthcare data unlike other kind of data has strict confidential, privacy and security concerns (Muir, 2011). HIPAA compliance is the most fundamental requirement when moving medical records to the cloud. Migrating entire data storages to a third party organization is not an easy task to do, especially when moving sensitive information such as healthcare data. Even more robust security should be assured because more concerns will arise with access controls, audit controls, authentication, authorization, transmission security and storage security in order to avoid exposing the information to unauthorized entities.

These issues are an obstacle that have slowed the cloud adoption and should be addressed in order to enable the trustworthiness of cloud systems. Fortunately, many of the biggest cloud providers in the market such as Microsoft, Google, and Amazon have commitments to develop the best policies and practices to secure a customer's data and privacy (Kuo, 2011).

\subsection{Interoperability}

Interoperability is one of the biggest challenges when moving healthcare systems to the cloud. It is due to the vast existence of different protocols, O.S, programming languages, platforms, data formats, databases and approaches that different healthcare organization have been using. Healthcare systems are not currently designed using common data modeling constructs resulting in different database designs (Myers, 2012) and incompatible systems.

Healthcare systems interoperability must occur in several different ways: at the provider, software, computer, data levels and system integration (Myers, 2012). Providers have generally maintained their own independent data and the incompatibility of healthcare systems largely prohibits its cross-institutional use (Myers, 2012). To embrace the cloud, health organizations must integrate their existing systems with modern web and cloud based systems (Muir, 2011). In addition, they should standardize processes such as the process of getting patients information and saving it to the cloud storage.

Computer software developers must not only cooperate but also share a common data model and design products that can interact with each other (Myers, 2012). Moreover, they must meet legal frameworks and standards in order to comply with law related with HIPAA requirements.

Data integration is fundamental and a necessary prerequisite to systems integration (Myers, 2012). In addition data standardization and formatting is another obstacle when designing cloud applications. For example, something as innocuous as a phone number may be formatted in one system as 1234567890 whereas another system will need (123) 456-7890, requiring software engineers to write custom code--costing time and money (Muir, 2011). Moreover, currently there is nothing that ties healthcare data together in a coherent and uniform way, which is expensive, redundant and insufficient (Myers, 2012). Therefore, integration and inter-operability can be reached by using universal standards, which makes version control, updating and maintenance easier.

A new approach to developing healthcare systems should be taken in order to design more interoperable systems. This change will result in numerous and substantial benefits to the health community. Integrating current healthcare systems and making them interoperable with the newest cloud-based development looks to be a challenging task. Although, by designing flexible and scalable standards and by integrating medical data will greatly benefit and assist the various caregivers.

\section{Conclusions}

The current trend of adopting cloud computing in the medical field can improve and solve several collaborative information issues in healthcare organizations as well as cost optimizations. Standardized cloud-based applications will bring obvious advantages to patients, physicians, insurance companies, pharmacies, imagining centers, etc. when sharing information across medical organizations yielding better results. Challenges such as security concerns and interoperability will rise due to the cloud-computing model. Therefore, the adoption of the cloud is progressing slowly. Through the implementation of best practices in the design, deployment and use of it will hopefully generate a future growth of the cloud-based systems adoption, despite all of the obstacles.

\section{Acknowledgement}

This research has been supported by the Fidelity National Financial Distinguished Professorship in Computer and Information Sciences.

\section{References}

Allen, S. (2011). Cloud Computing and Health Care Security. Cloud Computing Journal. Retrieved from http://cloudcomputing.sys-con.com/node/1796151 
Barry, J., \& Napatech. (2011). Testing the Cloud: Assuring Availability. Retrieved from http://www.hpcinthecloud.com/hpccloud/2011-08-16/testing_the_cloud:_assuring_availability.html

Blaisdell, R. (2012). Cloud benefits in the health Industry. Retrieved from http://www.cloudtweaks.com/2012/02/cloud-benefits-in-the-health-industry/

Cloud Security Alliance. (2010). Top Threats to Cloud Computing V1.0. Retrieved from https://cloudsecurityalliance.org/topthreats/csathreats.v1.0.pdf

Gullo, C. (2011). Half of doctors to use medical apps in 2012. Retrieved from http://mobihealthnews.com/14703/half-of-doctors-to-use-medical-apps-by-2012/

HealthImaging. (2010). Acumen Nabs ONC Cloud Computing Contract. Retrieved from http://www.healthimaging.com/index.php?option=com_articles\&view=article\&id=20648:acumen-nabs-onc -cloud-computing-contract\&division=hiit

Horowitz, B. (2011). Cloud Computing Brings Challenges for Health Care Data Storage, Privacy. Retrieved from

http://www.eweek.com/c/a/Health-Care-IT/Cloud-Computing-Brings-Challenges-for-Health-Care-Data-Sto rage-Privacy-851608/

Kabachinski, J. (2011). What's the forecast for cloud computing in healthcare? Biomedical Instrumentation \& Technology, 45(2), 146-150. http://dx.doi.org/10.2345/0899-8205-45.2.146

Korea IT Times. (2010). Telstra Plans Launch of E-Health Cloud Services, Tip of the Iceberg for Opportunity. Retrieved from http://www.koreaittimes.com/story/9826/telstra-plans-launch-e-health-cloud-services-tip-iceberg-opportunit $\mathrm{y}$

Kuo, A. M. (2011). Opportunities and Challenges of Cloud Computing to Improve Health Care Services. Journal of Medical Internet Research, 13(3), e67. http://dx.doi.org/10.2196/jmir.1867

Kupferman, J., Silverman, J., Jara, P., \& Browne, J. (2009). Scaling Into the Cloud. Retrieved from http://cs.ucsb.edu/ jkupferman/docs/ScalingIntoTheClouds.pdf

Kuttikrishnan, D. (2011). Cloud Computing: The road ahead. Retrieved from $\mathrm{http} / /$ www.datamation.com/cloud-computing/cloud-computing-the-road-ahead-1.html

Logic Monitor. (2012). LogicMonitor: ARCHITECTURE WHITE PAPER. Retrieved from http://www.logicmonitor.com/downloads/Architecture.pdf?84cd58

Microsoft. (2012). Microsoft HealthVault. $\quad$ Retrieved from http://www.microsoft.com/en-us/healthvault/organize/medical-records.aspx, Last retrieved 2012

Muir, E. (2011). Challenges of cloud computing in healthcare integration. Retrieved from http://www.zdnet.com/news/challenges-of-cloud-computing-in-healthcare-integration/6266971

Myers, J. E. (2012). Data Modeling for Healthcare Systems Integration: Use of the MetaModel. Retrieved from http://www.metadata.com/whitepapers/myers1.pdf

Pandora. (2011). FMS-Virtualization and cloud computing monitoring. Retrieved from http://pandorafms.com/downloads/PandoraFMS_Virtual_Enviroment_Monitoring.pdf

Rackspace Hosting. (2011). High Availability Cloud Environments. Retrieved from http://www.codeproject.com/Articles/157992/High-Availability-Cloud-Environments

Raut, V. (2011). Cloud Computing and Health Care. Cloud Computing Journal. Retrieved from $\mathrm{http}: / /$ cloudcomputing.sys-con.com/node/2026409

Rui, Z., \& Ling, L. (2010). Security Models and Requirements for Healthcare Application Clouds. Cloud Computing (CLOUD), 2010 IEEE 3rd International Conference.

Shimrat, O. (2009). Cloud Computing and Healthcare. Technology Matters. Retrieved from http://www.himss.org/content/files/Code\%2093_Shimrat_CloudComputingandHealthcare_2009.pdf

Terry, K. (2012). Cloud computing in healthcare: the question is not if, but when. Retrieved from $\mathrm{http}: / / w w w . f i e r c e h e a l t h i t . c o m / s t o r y /$ cloud-computing-healthcare-question-not-if-when/2012-01-09

Violino, B. (2011). Accenture, AT\&T Offer Cloud-Based Medical Imaging. Retrieved from $\mathrm{http} / / / \mathrm{www}$.information-management.com/news/Accenture-ATT-cloud-computing-medical-imaging-solutio 
n-10021574-1.html

Wan, D., Greenway, A., Harris, J. G., \& Alter, A. E. (2010). Six questions every health industry executive should ask about cloud computing. Retrieved from http://www.accenture.com/SiteCollectionDocuments/PDF/Accenture_Cloud_Healthcare_PoV.pdf

Wang, X. (2010). Application of Cloud Computing in the Health Information System. Computer Application and $\begin{array}{llll}\text { System } & \text { Modeling } & \text { Retrieved } & \text { from }\end{array}$ http://ieeexplore.ieee.org/stamp/stamp.jsp?tp=\&arnumber=5619051

Webb, G. (2012). Making the cloud work for healthcare, Health Management Technology. Retrieved from http://connection.ebscohost.com/c/articles/71285402/making-cloud-work-healthcare

Whitemore, J. (2012). Five key considerations for healthcare facilities before moving to the cloud. Retrieved from http://www.mhimss.org/news/five-key-considerations-healthcare-facilities-moving-cloud 